\title{
Surgical Research
}

\section{Intestinal Obstruction in an 11-Month-Old Infant following Ingestion of a Superabsorbent Polymer}

\section{Mirian Ramos-Mercado', Wihelma Echevarria', Aurelio Segundo², Priyanka Bajaj' and Jorge Perez ${ }^{1 *}$}

\author{
'San Juan Bautista School of Medicine, Expreso Luis A. Ferré, \\ Caguas, P.R., USA. \\ ${ }^{2}$ HIMA San Pablo Hospital, 100 Avenida Luis Muñoz Marín, \\ Caguas, USA.
}

\section{*Correspondence:}

Jorge Perez, MD, Associate Professor, San Juan Bautista School of Medicine, Expreso Luis A. Ferré, Caguas, P.R., USA, E-mail: jperez@sanjuanbautista.edu.

Received: 19 June 2019; Accepted: 21 July 2019

Citation: Mirian Ramos-Mercado, Wihelma Echevarria, Aurelio Segundo, et al. Intestinal Obstruction in an 11-Month-Old Infant following Ingestion of a Superabsorbent Polymer. Surg Res. 2019; 1(1): 1-3.

ABSTRACT
Foreign body ingestion comprises one of the most common pediatric complaints in the emergency department.
While most foreign bodies pass spontaneously through the gastrointestinal tract, water-retaining agents carry a
higher risk of complications. We report a case of ingestion of a superabsorbent polymer ball by an 11-month-old
infant who developed small bowel obstruction requiring laparotomy and resection.

\section{Keywords}

Accidental ingestion, Bowel obstruction, Expanding foreign body, Gastrointestinal tract, Superabsorbent polymer.

\section{Introduction}

In early childhood, foreign body ingestion often occurs due to instinctive oral exploration of the surrounding environment and comprises one of the most common pediatric complaints in the emergency department [1,2]. Most events occur in children between 6 months and 3 years of age [3]. It has been reported that $40 \%$ of foreign body ingestions in children are unwitnessed [4]. Fortunately, most foreign bodies, particularly smooth and round objects, pass spontaneously through the gastrointestinal tract without posing significant threats [1]. Most ingestions are asymptomatic; however, some carry a higher risk of complications [4,5]. Approximately 10-20\% of ingested foreign bodies are recovered endoscopically and fewer than $1 \%$ require surgical removal [5]. We report a case of an 11-month-old infant who ingested a superabsorbent polymer (SAP) ball leading to small bowel obstruction requiring laparotomy and resection of the affected segment.

\section{Case Report}

An 11-month old female infant presented to the hospital with a history of bilious vomiting. Abdominal X-rays demonstrated significant gaseous distention of loops of bowel in the upper abdomen (Figure 1). Chest X-rays revealed bilateral prominent reticulonodular opacities.

The child was admitted and, on hospital day three, subsequent abdominal X-rays showed dilatation of small bowel loops, suggestive of a potential mechanical obstruction. The patient had developed regional ileus and persistent bilious vomiting, warranting additional workup.

Abdominopelvic CT scans without contrast identified small bilateral pleural effusions. Upon instillation into the stomach, oral contrast was seen within the antrum to the first, second and third portion of the duodenum. The scans revealed severe diffuse dilatation of multiple small bowel loops involving the jejunum and ileum that measured up to $3.6 \mathrm{~cm}$ in diameter with a mild degree of wall thickening and multiple air-fluid levels (Figure 2). Furthermore, collapse of the distal ileum and the entire colon in this patient raised suspicion for intestinal obstruction of moderate to high degree, considering that the normal diameter of the small bowel ranges from 2.5-3.0 $\mathrm{cm}$ [4]. Metabolic panel revealed decreased BUN and creatinine levels in the patient. Mild right hydronephrosis and underdistention of most of the bladder were noted, likely from ureter compression at the pelvic brim by the dilated bowel loops. Overall distention of the abdominal wall was present.

Surgical intervention was subsequently recommended. At exploratory laparotomy, a foreign object was found in the distal ileum (Figure 3). Upon resection, a superabsorbent polymer (SAP) 
ball was found adherent to the affected segment (Figure 4).

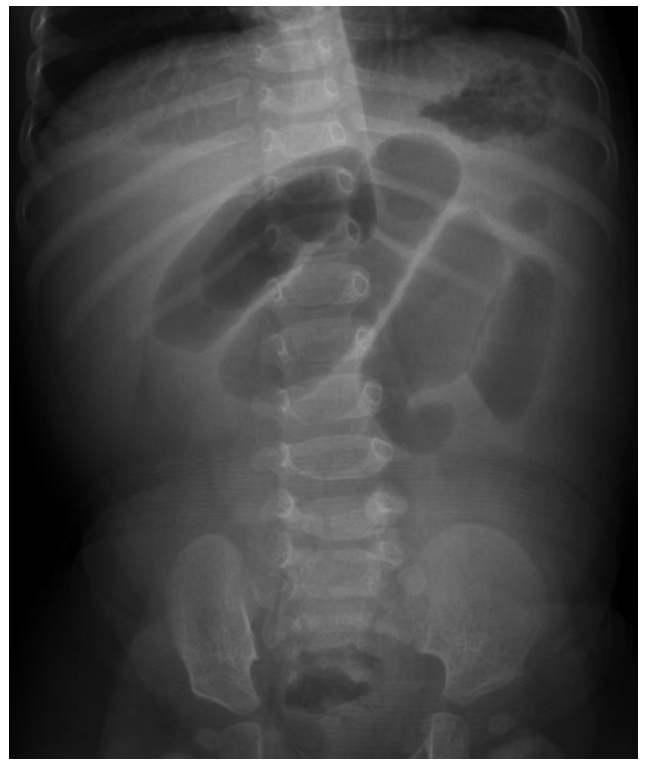

Figure 1: Abdominal $\mathrm{X}$ ray demonstrating gaseous distention of loops in the upper abdomen.
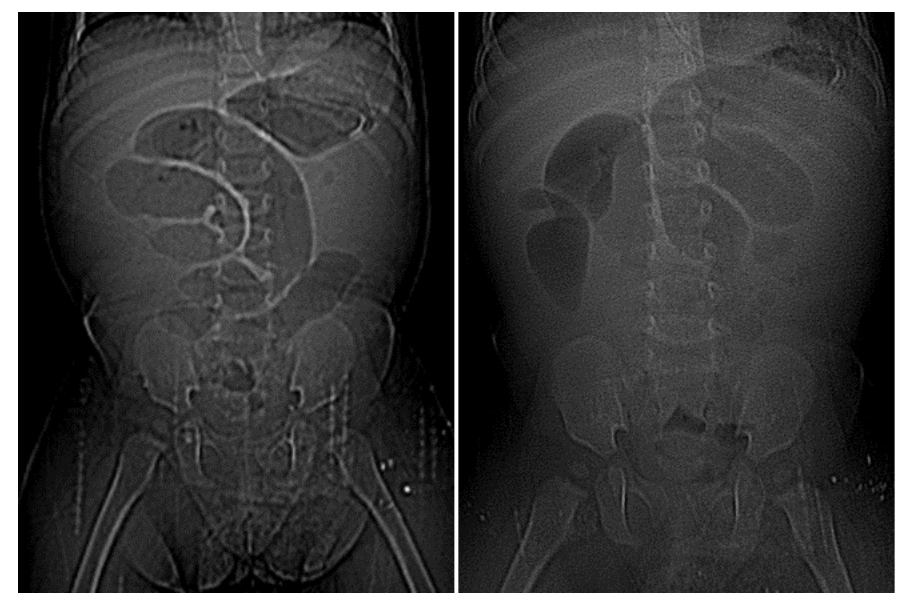

Figure 2: Abdominopelvic CT scans showing severe diffuse dilatation of multiple small bowel loops.

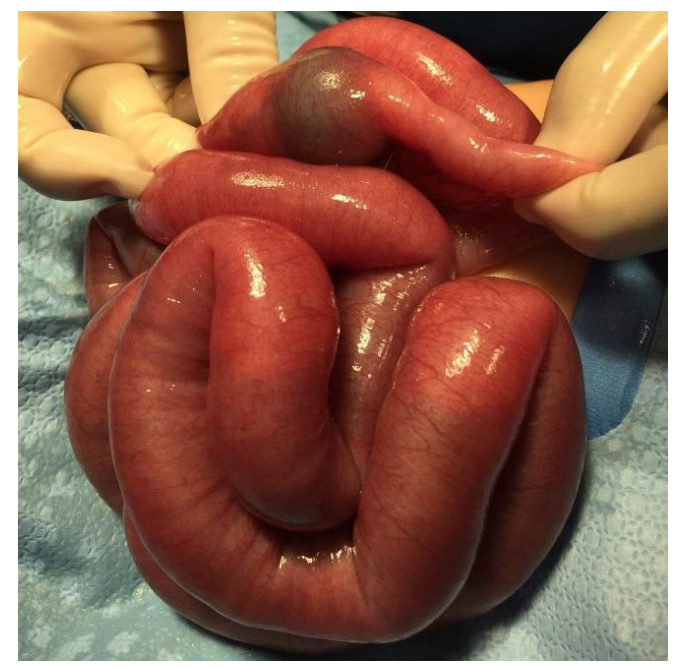

Figure 3: Exploratory laparotomy revealing a foreign object in the distal ileum.

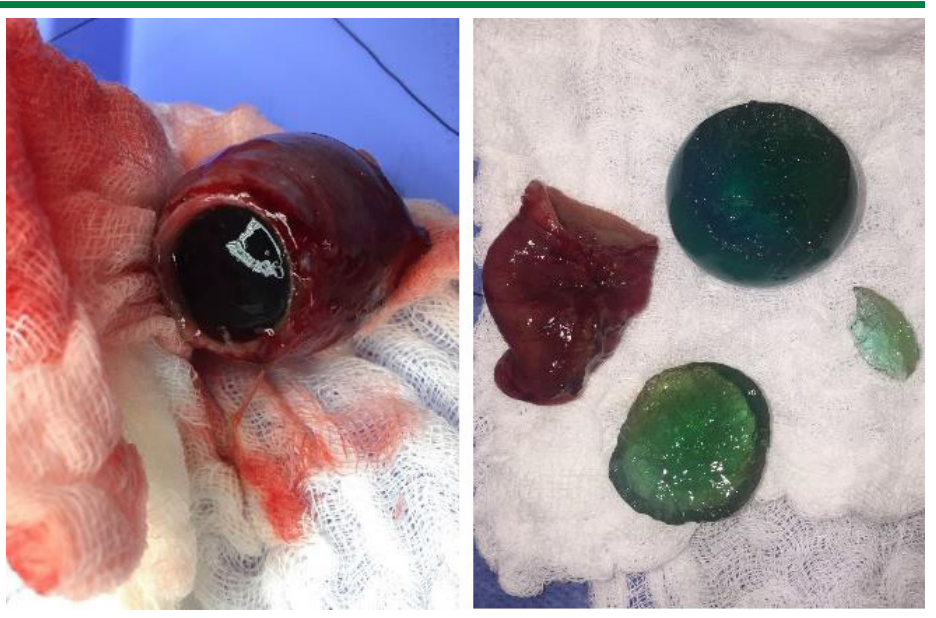

Figure 4: A SAP ball adhering to the affected intestinal segment.

Abdominal X-rays on postoperative day one revealed mild dilatation of the small and large bowel loops, consistent with postsurgical ileus. On postoperative day four, a portable babygram confirmed decreased distention and thickness of bowel loops (Figure 5).

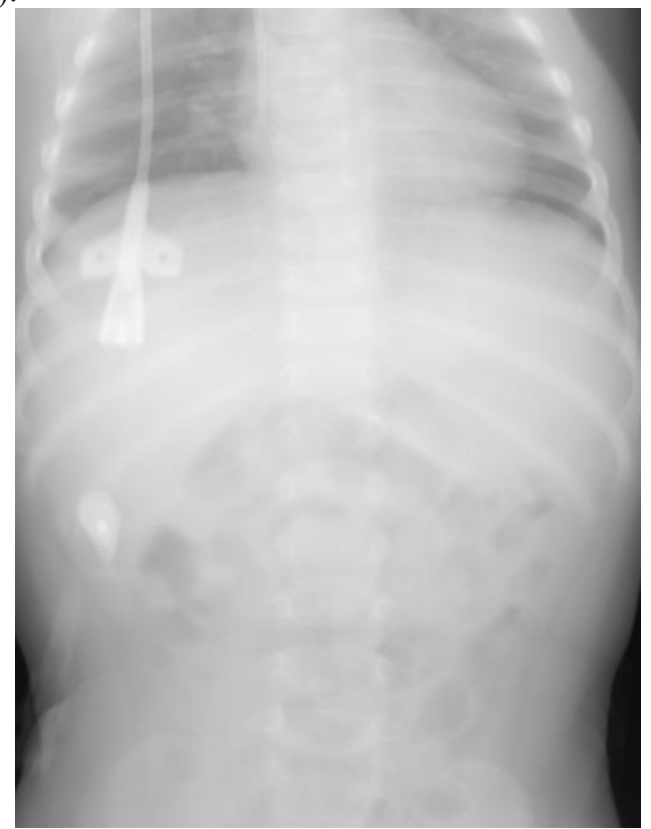

Figure 5: Portable babygram confirming decreased distention and thickness of bowel loops.

\section{Discussion}

Superabsorbent polymer (SAP), also known as polyacrylamide, polymethyl acrylate and sodium polyacrylate, is a water-retaining agent that absorbs large quantities of fluid [4,5]. SAP balls and beads are commonly used in agriculture; gardening; flower displays; supplying water to small animals and insects; absorbent fillers inside disposable diapers; containment of flooding; and, children's toys [5]. Specific SAP compositions are not readily available and whether distinct compositions respond differently in terms of water absorption and diameter expansion is unknown [5].

Many of these balls have gained media attention in the last decade 
as their ingestion may lead to potential complications due to their swelling properties in liquids [4,6]. Their small size makes them easy to swallow initially before expanding in transit through the gastrointestinal tract [5]. Additionally, they are often brightly colored and may appear candy-like to children [4].

In 2012 and 2013, deaths of children overseas and injuries in the United States prompted the Consumer Product Safety Commission (CPSC) to issue a voluntary recall of several types of waterabsorbing balls, beads and toys [7]. One such toy was WaterBalz, advertised to grow up to 400 times its original size after submersion in water $[4,5]$. One case reported that ingestion of this bead caused complete intestinal obstruction in an 8-month-old infant, similarly warranting a diagnostic laparoscopy and enterotomy [4].

Complications following ingestion of SAP-containing balls may range from partial to complete intestinal obstruction. 8 Six cases with complications involving small bowel obstruction have been reported to date $[2,4,8-11]$. The ages of patients who underwent surgery were between 6 and 18 months old. One 6-month-old patient in Pakistan died due to postoperative sepsis [9]. When comparing these cases with other uncomplicated cases, it was determined that infants under 18 months should be observed more closely [1]. Symptoms of intestinal blockage include refusing to eat; complaints that something is stuck in the chest; drooling; vomiting; wheezing; constipation; and, abdominal pain, swelling or soreness [7].

SAP balls pose a peculiar clinical problem as their ingestion is often difficult to diagnose. Unlike radiopaque foreign bodies that can be detected on plain radiographs, SAP balls are radiolucent and not visible on X-rays [1,12].

Obstruction should be identified by axial imaging or abdominal ultrasounds [4]. An in-vitro analysis of the previously mentioned WaterBalz toy demonstrated that the SAP-containing bead was small enough to pass through the pylorus within 2 to 4 hours of ingestion; however, its growth could exceed the normal diameter of the small bowel within 6 hours [4]. Accordingly, witnessed ingestions of SAP balls should be followed by timely endoscopic removal before passing the pylorus to prevent further bowel obstruction [3]. Researchers have concluded that polyethylene glycol (PEG) administered to promote intestinal transit of SAPcontaining beads may paradoxically result in increased bead diameter [5]. Therefore, if unable to be identified on endoscopy, surgical intervention should be considered as there is a high likelihood that nonoperative management will fail [5].

Toys that incorporate SAP balls are currently banned in the United Kingdom, Malaysia and Italy [10]. Previous reports of pediatric ingestion and recall of several types of water-absorbing balls prompted the CPSC to issue changes in its mandatory toy standard ASTM F963-16, which are in effect as of April 2017 [13]. These include safety requirements and testing methodologies designed to address the public health hazard posed by expanding materials. Currently, the safety requirements approve objects measuring $2 \mathrm{~cm}$ or less in diameter for viable passage through the pyloric sphincter.13 However, additional research is needed to determine if objects ranging from $1-2 \mathrm{~cm}$ in diameter are likely to cause blockage of a young child's small intestine or ileocecal sphincter.

This report should serve to increase awareness for medical practitioners, parents and caretakers surrounding the risks that accompany accidental ingestion of SAP products. Parents and caretakers should be cognizant of the existence of such products in the market and medical practitioners should be prepared to intervene in a timely manner.

\section{Patient Consent}

Consent to publish the case report was not obtained. This report does not contain any personal information that could lead to the identification of the patient.

\section{References}

1. Mehmetoğlu F. Aerodigestive foreign bodies in an infant: case report and literature review. Firat Medical Journal. 2016; 21: 224226.

2. Hao D.Pham, Lesli A, Taylorb. Small bowel obstruction due to ingested superabsorbent beads. Journal of Pediatric Surgery Case Reports. 2015; 3: 190-191.

3. Ji Hyuk Lee. Foreign Body Ingestion in Children. Clinical Endoscopy. 2018; 51: 129-136.

4. Irving J. Zamora, Lan T. Vu, Emily L. Larimer, et al. WaterAbsorbing Balls: A "Growing" Problem. Pediatrics. 2012; 130:1011-1014.

5. Darracq MA, Cullen J, Rentmeester L, et al. The Magic Water Absorbing Bead - Risk of Pediatric Bowel Obstruction? Pediatric Emergency Care. 2015; 31: 416-418.

6. Feride Mehmetoğlu. Retrospective 10-Year Analysis of Water Absorbent Bead Ingestion in Children. Emergency Medicine International. 2018; 2018: 1-4.

7. https://www.aappublications.org/news/2016/03/22/ WaterBeads032216

8. Mirza B, Ijaz L, Sheikh A. Decorative crystal balls causing intestinal perforation. Journal of Indian Association of Pediatric Surgeons. 2011; 16: 106-107.

9. Mirza B, Sheikh A. Mortality in a Case of Crystal Gel Ball Ingestion: An Alert for Parents. Association of Pediatric Surgeons of Pakistan Journal of Case Reports. 2012; 3: 6.

10. Muthukumaran J, Vivek S. Intestinal Obstruction due to Accidental Ingestion of Hygroscopic Foreign Body. Indian Pediatrics. 2014; 51: 1022-1023.

11. Moon JS, Bliss D, Hunter CJ. An unusual case of small bowel obstruction in a child caused by ingestion of water-storing gel beads. Journal of Pediatric Surgery. 2012; 47: 19-22.

12. Jackson J, Randell KA, Knapp JF. Two Year Old With Water Bead Ingestion. Pediatric Emergency Care. 2015; 31: 605-607.

13. Proposed Revision of ASTM F963 Mandatory Toy Standard. United States Consumer Product Safety Commission. 2017. 